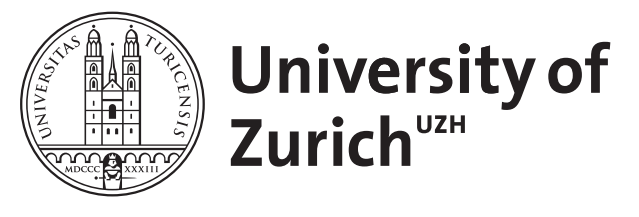

\title{
She's the boss: signaling in pollen tube reception
}

\author{
Kessler, S A ; Grossniklaus, U
}

\begin{abstract}
In angiosperms, the sperm cells are carried within the pollen tubes (male gametophytes) to the female gametophyte so that double fertilization can occur. The female gametophyte exerts control over the male, with specialized cells known as synergids guiding the pollen tubes and controlling their behavior when they enter the female gametophyte so that the sperm cells can be delivered to the egg and central cell. Upon pollen tube arrival at the ovule, signal transduction cascades mediated by receptor-like kinases are initiated in both the synergid and the tip of the pollen tube, leading to synergid cell death and pollen tube rupture. In this review, we discuss the role of these receptors and of newly discovered members of the pollen tube reception pathway.
\end{abstract}

DOI: https://doi.org/10.1016/j.pbi.2011.07.012

Posted at the Zurich Open Repository and Archive, University of Zurich

ZORA URL: https://doi.org/10.5167/uzh-54525

Journal Article

Accepted Version

Originally published at:

Kessler, S A; Grossniklaus, U (2011). She's the boss: signaling in pollen tube reception. Current Opinion in Plant Biology, 14(5):622-627.

DOI: https://doi.org/10.1016/j.pbi.2011.07.012 


\section{She's the Boss: Signaling in Pollen Tube Reception}

Sharon A. Kessler $^{1 *}$ and Ueli Grossniklaus ${ }^{1}$

${ }^{1}$ Institute of Plant Biology \& Zürich-Basel Plant Science Center, University of Zürich, Zollikerstrasse 107, CH-8008 Zürich, Switzerland

$\begin{array}{ll}\text { Corresponding author: } & \text { Sharon A. Kessler } \\ & \text { Institute of Plant Biology } \\ & \text { University of Zürich } \\ & \text { Zollikerstrasse 107 } \\ & \text { CH-8008 Zürich } \\ & \text { Tel: +41 44 634 } 8259 \\ & \text { Fax: }+41446348204 \\ & \text { Email: skessler@ botinst.uzh.ch }\end{array}$

Key words: pollen tube reception, female gametophyte, plant defense, receptor-likekinase, FERONIA, cysteine-rich peptide, Arabidopsis thaliana, Torenia fournieri, maize 


\begin{abstract}
In angiosperms, the sperm cells are carried within the pollen tubes (male gametophytes) to the female gametophyte so that double fertilization can occur. The female gametophyte exerts control over the male, with specialized cells known as synergids guiding the pollen tubes and controlling their behavior when they enter the female gametophyte so that the sperm cells can be delivered to the egg and central cell. Upon pollen tube arrival at the ovule, signal transduction cascades mediated by receptor-like kinases are initiated in both the synergid and the tip of the pollen tube, leading to synergid cell death and pollen tube rupture. In this review, we discuss the role of these receptors and of newly discovered members of the pollen tube reception pathway.
\end{abstract}




\section{Introduction}

A major trend during land plant evolution was the extreme reduction of the gametophytic phase of the life cycle. The female gametophyte (embryo sac) became embedded in female sporophytic tissue, while the male gametophyte was reduced to a three-celled pollen grain containing a vegetative cell harboring two sperm cells that are protected from the environment. Since these sperm cells are non-motile, the evolution of pollen grains went hand-in-hand with the evolution of new mechanisms of fertilization. In seed plants, the sperm cells are carried to the female gametophyte in tip-growing pollen tubes (PTs), such that an aqueous environment is no longer necessary for fertilization (syphonogamy). Consequently, male gametes could be dispersed over long distances, with pollen carried by wind or various pollinators. The evolution of fast growing PTs appears to be a key life history trait that was important for angiosperm diversification [1]. Along with the evolution of reduced gametophytes came the necessity for the female to (1) distinguish between self and non-self pollen grains in order to prevent wasted resources in unsuccessful pollination attempts, and (2) to control the growth of the PT so that it discharges the sperm at the appropriate time and place for double fertilization to occur.

In angiosperms, pollination requires communication between the male gametophyte and both the sporophytic and gametophytic tissues of the female (Figure 1). Pollen lands on a receptive stigma and germinates to produce a tip-growing PT that grows towards the style and into the transmitting tract. Many species have a selfincompatibility system that acts during these early stages of pollination and prevents self-fertilization [2]. PTs grow between the cells of the transmitting tract to reach the ovary, where they exit the transmitting tract and are attracted to the ovules by a signal produced by the female gametophyte [3]. The molecular basis of PT guidance to the 
ovule is reviewed by Higashiyama (this issue). In processes collectively termed PT reception, one PT per ovule enters the female gametophyte through a synergid cell, stops growth and ruptures to release the two sperm cells. One sperm fuses with the egg to form the embryo and the other with the central cell to form the triploid endosperm in a process known as double fertilization. This review will focus on recent progress in understanding the signaling events between the male and female gametophytes during PT reception.

\section{The synergid cells: gateway to the female gametophyte}

The synergid cells of the female gametophyte control the final stages of PT attraction and reception. Synergid cells flank the egg cell at the micropylar end of the female gametophyte. They have a specialized structure, with discontinuous cell walls near the egg and central cell boundaries and a micropylar domain known as the filiform apparatus, which has a thick cell wall and finger-like projections into the synergid cytoplasm (reviewed in [4]). Laser ablation experiments in Torenia fournieri showed that at least one viable synergid cell is necessary for PT attraction [5]. In Arabidopsis thaliana, the myb98 mutant has a defective filiform apparatus and also fails to attract PTs, indicating that proper synergid differentiation is also necessary for PT attraction in other species [6]. Further PT attraction experiments in Torenia showed that a species-specific, diffusible signal is emitted from the synergids to attract the PT [7]. Small cysteine rich proteins (CRPs) have been implicated in many different phases of pollen-pistil interactions, from self-incompatibility early in pollination, to PT growth and guidance (reviewed in [8,9]). In Torenia, CRPs called LUREs were shown to be secreted toward the micropylar ends of synergids and localized in the filiform apparatus in order to mediate short-range micropylar PT 
guidance $\left[10^{\circ}\right.$ ]. A role for LURE-like CRPs in PT guidance in other species has not been shown. However, CRPs were also identified to be highly expressed in maize synergid cells [11] and another short, secreted protein, ZmEA1, was shown to be involved in micropylar PT guidance in maize [12].

\section{Pollen tube entry into the synergid cells}

Upon arrival at the micropylar end of the ovule, the PT enters the female gametophyte through one of the synergid cells. The PT grows through the filiform apparatus, stops growth, and ruptures to release the two sperm cells so that double fertilization can occur. The synergid cell that receives the PT undergoes cell death either before or upon entry of the PT $[13,14]$. This cell death is thought to be important for facilitating the fertilization process, as Arabidopsis mutants like gfa2 with defective synergid cell death remain unfertilized [15], but its exact role remains unclear. It is clear that synergid cell death is a highly controlled and coordinated process as the synergid plays an active role in the developmental events immediately prior to gamete fusion. Synergid cell death could be important for allowing the PT to enter the synergid, e.g., by reducing its turgor pressure to allow the explosive discharge of the sperm cells [16], and/or for setting up an environment which allows PT reception and the delivery of the sperm cells to the egg and central cell $[17,18]$. Whether synergid cell death is a cause or a consequence of PT rupture remains controversial in Arabidopsis. Sandaklie-Nikolova, et al. [19] showed that synergid degeneration scored by loss of GFP marker expression in the cytoplasm and change in synergid shape occurred after PT arrival at the female gametophyte but more than 100

minutes before PT discharge. In contrast, a recent report from Hamamura, et al. [20 ] showed that loss of a nuclear-expressed synergid GFP marker occurred 
simultaneously with PT discharge, suggesting that breakdown of the receptive synergid is dependent on PT discharge. The latter hypothesis is supported by the finding that the sirène (srn) allele of the feronia (fer) mutation and lorelei-5 (lre-5) both have defects in PT rupture (see next section) and do not exhibit normal synergid cell death [16,21]. In addition, the abstinence by mutual consent (amc) mutant has both PT reception and synergid cell death defects when the mutation is present in both male and female gametophytes. This indicates that this peroxin gene may be necessary for cell death in both the PT and the synergid, perhaps by modulating the production of reactive oxygen species (ROS) [22]. On the other hand, ultrastructural analysis of fer embryo sacs indicate that the penetrated synergid displays electrondense material [23], which is typical of the degenerating synergid [24]. However, this may be a very late maker for synergid cell death and all current methods for detecting synergid degeneration rely on indirect observations (i.e. loss of GFP signal and fixation/staining protocols that detect late stages of cell death). A definitive analysis of the role of synergid degeneration in PT reception awaits the development of more sophisticated tools for monitoring early stages of cell death in this specialized cell type.

\section{Signaling during PT reception: female components}

The first evidence that PT reception is under the control of the female gametophyte came from the identification of mutants in which PT attraction to the ovules was normal, but PTs failed to stop growing and rupture within the receptive synergid cell (Figure 1). The first two mutants identified were fer and $\operatorname{srn}[16,23]$, which were later found to be alleles of the same gene [25], hereafter referred to as FER. fer mutants have normal differentiation of the female gametophyte, but are 
semi-sterile, with around $50 \%$ unfertilized ovules in heterozygous plants due to PT overgrowth within the synergids and a lack of PT rupture. Reciprocal crosses between fer/FER and wild-type plants showed that while fer PTs undergo normal reception in wild-type ovules, wild-type PTs exhibits overgrowth, and fail to stop growth and discharge the sperm cells in fer ovules. This indicated that fer is a female gametophytic mutation [23]. FER encodes a receptor-like kinase of the CrRLK1L-1 subfamily of kinases. In synergid cells, FER protein is concentrated at the filiform apparatus, leading to the hypothesis that FER is responsible for sensing PT arrival and triggering a signal transduction cascade in the synergid that feeds back to the PT, triggering PT rupture [25].

Other female components of the PT reception pathway in Arabidopsis include a glycosylphosphatidylinositol[21,26,27] (GPI)-anchored protein, LORELEI (LRE), and a synergid-specific Mildew Resistance Locus O (MLO)-like protein, NORTIA (NTA) . Like fer, lre and $n t a$ mutants both have unfertilized ovules due to a failure in PT reception. Since mutant alleles are (rarely) transmitted through the female gametophyte, homozygous mutants can be recovered, albeit at low frequencies for some of the mutants. In contrast to fer/fer mutants which have $>90 \%$ unfertilized ovules [27*0], the frequency of unfertilized ovules in lre/lre and nta/nta mutants ranges from $\sim 80 \%$ in lre alleles [21] down to $\sim 20 \%$ in $n t a$ alleles [27"*], indicating that while FER activity is necessary for signaling between the synergid and PT, other, yet unknown factors act redundantly with LRE and NTA.

The link between FER, NTA, and LRE remains unknown, although the similarity in the mutant phenotypes suggests that these three proteins act in the same pathway. In fact, recent studies show NTA to be downstream of FER signaling (see below). All three proteins belong to plant-specific gene families and are predicted to 
be associated with the plasma membrane. FER has a signal peptide and a transmembrane domain and has been shown to localize to the plasma membrane in leaf epidermal cells and to be concentrated in the plasma membrane of the filiform apparatus before PT reception [25]. NTA has a signal peptide, 7 predicted transmembrane domains, and a calmodulin-binding domain at its C-terminus. While NTA is capable of being targeted to the plasma membrane when transiently expressed in onion epidermal cells, in synergid cells, an NTA-GFP fusion that complements the mutation is localized in the cytoplasm (possibly the endomembrane system) before PT arrival, but it becomes polarly localized to the filiform apparatus when the PT arrives at the female gametophyte. In fer mutant ovules, NTA-GFP does not relocalize to the micropylar end of the synergid, indicating that the FER pathway is necessary for the relocalization of NTA $\left[27^{\circ}\right]$. LRE has a predicted signal peptide and a putative GPI anchor domain in its C-terminus, leading to the hypothesis that LRE is transported to the plasma membrane through the secretory system and is attached to the outside of the synergid cell by the GPI anchor. An LRE-GFP fusion protein was detected on the surface of transfected Arabidopsis mesophyll protoplasts, but the localization of LRE protein in synergid cells has not been determined due to difficulties in obtaining stably transformed plants that express fluorescently-tagged LRE [26].

While FER, LRE, and NTA-related genes are found throughout the plant kingdom, whether the FER-mediated signaling pathway is conserved in species besides Arabidopsis remains an open question. In recent years, maize has emerged as a model system for studying pollination in the grasses (reviewed in [28]). While functional studies of the role of FER, NTA, and LRE have not been reported in maize, this system is now being used to discover new components of the PT-reception pathway. RNA-interference of a defensin-like gene known as ZmES4 results in a fer- 
like PT overgrowth phenotype [29³. Like NTA [27], ZmES4 seems to be stored in the secretory system before fertilization. Interestingly, chemically-synthesized ZmES4 applied to in vitro-germinated PTs led to plasma membrane depolarization and bursting in a species-specific manner [29“]. This indicates that, at least in maize, ZmES4 may act as a direct mediator of PT discharge at the end of the PT reception signal-transduction cascade.

\section{Signaling during PT reception: male components}

While forward genetic screens have succeeded in identifying female components of the PT reception pathway, identification of male components has proven to be much more difficult, and so far only candidate genes have been identified. ANXUR1 (ANX1) and ANXUR2 (ANX2) are closely related pollenexpressed FER-like receptor-like kinases [30“31“*]. anxl/anx2 double mutant PTs rupture prematurely in the transmitting tract of the style, indicating that these RLKs are responsible for maintaining PT integrity during tip growth. Thus, ANX1/ANX2 might function to control PT bursting inside the synergids, receiving a signal from the synergid cell, leading to their deactivation to allow the PT to burst [30“].

A second candidate for controlling PT bursting in the synergid cells came from studies of the ZmES4 peptide in maize [29*3. The structural similarity of ZmES4 to invertebrate venom peptides that have been shown to modulate either $\mathrm{K}^{+}$or $\mathrm{Na}^{+}$ion channels led the authors to test the response of different ion channels to application of ZmES4. This led to the identification of KZM1, a PT-expressed shaker $\mathrm{K}^{+}$channel, which opens in response to ZmES4 application [29“*]. These experiments were done in a heterologous system (Xenopus laevis oocytes) and should be explored more in planta, but they nevertheless provide an intriguing hypothesis that at PT 
reception, ZmES4 is released from the secretory system of the synergid cell and binds to KZM1 on the PT, triggering $\mathrm{K}^{+}$influx that leads to bursting of the PT and the release of sperm cells. Interestingly, an ion channel has also been implicated in Arabidopsis PT bursting. Mutants in the $\mathrm{Ca}^{2+}$ pump ACA9 attract PTs that enter the synergids and stop growing, but do not burst [32]. Thus, the balance of ion gradients in PTs and synergid cells may play a critical role in controlling PT discharge.

\section{Conclusions}

Even though the elucidation of the complete signal transduction cascade between the synergid cell and the PT during PT reception is far from being completed, the last few years have seen the discovery of several players on both the female and male sides. A common theme that emerged from the discovery of defensin-like genes involved in PT attraction and reception [10,29] was that higher plant reproduction may have taken advantage of defense pathways that already existed early in the evolution of land plants to evolve new, gametophyte-specific mechanisms for communication between the male and female [8]. This theme has continued to be supported by the recent finding that PT reception shares common components with the MLO-mediated powdery mildew susceptibility pathway, with NORTIA, a member of the MLO family of 7 membrane proteins playing a specific role in PT reception, and FERONIA having dual roles in both powdery mildew susceptibility and PT reception [27"*]. Powdery mildew hyphae and PTs are both tip growing cells that must penetrate another cell, FER seems to mediate both of these processes and may be important for controlling cell death. The discovery of the GPIanchored protein (GAP), LORELEI as a component of the PT reception machinery 
[26] is also reminiscent of disease responses, as another plant GAP, NDR-1 is involved in signal transduction in bacterial disease resistance pathways [33].

A rough framework can now be made for the intercellular signaling pathways that occur during PT reception (Figure 2). As a PT enters the receptive synergid cell, the FER RLK perceives a signal and triggers the relocalization of NTA to the filiform apparatus. LRE's exact role is unknown but it may act as part of the FER complex, or it could be involved in signaling back to the PT. At the tip of the PT, the ANX1/ANX2 RLKs could be involved in receiving a signal from the synergid, which then leads to growth arrest and rupture of the PT. The signal from the synergid back to the PT could be ZmES4, which also activates the KZM1 $\mathrm{K}^{+}$channel to trigger PT bursting.

Future challenges are to determine the connections between these proteins and to identify new components such as the ligands of FER and ANX1/ANX2. A recently discovered malectin-like carbohydrate domain in the extracellular domains of FER, ANX1, and ANX2 indicates that the ligand may be a carbohydrate released from the cell wall, or a protein with specific carbohydrate modifications [ $\left.34^{\circ}\right]$. While most angiosperms have synergid cells that function in PT reception, some species have evolved other mechanisms. It will be interesting to look at double fertilization in species that do not have synergids cells, such as Plumbago zeylandica [35], or in species where the PTs do not enter through the micropyle but rather through the chalaza, e.g in the genus Casuarina ([36]; reviewed in [37]). 
References and recommended reading

1. Williams JH: Novelties of the flowering plant pollen tube underlie diversification of a key life history stage. Proc Natl Acad Sci U S A 2008, 105:11259-11263.

2. Takayama S, Isogai A: Self-incompatibility in plants. Annu Rev Plant Biol 2005, 56:467-489.

3. Hulskamp M, Schneitz K, Pruitt RE: Genetic Evidence for a Long-Range Activity That Directs Pollen Tube Guidance in Arabidopsis. Plant Cell 1995, 7:57-64.

4. Higashiyama T: The synergid cell: attractor and acceptor of the pollen tube for double fertilization. J Plant Res 2002, 115:149-160.

5. Higashiyama T, Yabe S, Sasaki N, Nishimura Y, Miyagishima S, Kuroiwa H, Kuroiwa T: Pollen tube attraction by the synergid cell. Science 2001, 293:1480-1483.

6. Kasahara RD, Portereiko MF, Sandaklie-Nikolova L, Rabiger DS, Drews GN: MYB98 is required for pollen tube guidance and synergid cell differentiation in Arabidopsis. Plant Cell 2005, 17:2981-2992.

7. Higashiyama T, Inatsugi R, Sakamoto S, Sasaki N, Mori T, Kuroiwa H, Nakada T, Nozaki H, Kuroiwa T, Nakano A: Species preferentiality of the pollen tube attractant derived from the synergid cell of Torenia fournieri. Plant Physiol 2006, 142:481-491.

8. Dresselhaus T, Marton ML: Micropylar pollen tube guidance and burst: adapted from defense mechanisms? Curr Opin Plant Biol 2009, 12:773-780.

9. Higashiyama T: Peptide signaling in pollen-pistil interactions. Plant Cell Physiol 2010, 51:177-189.

-10. Okuda S, Tsutsui H, Shiina K, Sprunck S, Takeuchi H, Yui R, Kasahara RD, Hamamura Y, Mizukami A, Susaki D, et al.: Defensin-like polypeptide LUREs are pollen tube attractants secreted from synergid cells. Nature 2009, 458:357-361.

This paper reinforces the role of the synergids in the female control of PT behavior. LURE polypeptides from the synergids of Torenia fournieri are shown to act as species-specific PT attractants.

11. Cordts S, Bantin J, Wittich PE, Kranz E, Lorz H, Dresselhaus T: ZmES genes encode peptides with structural homology to defensins and are specifically expressed in the female gametophyte of maize. Plant $J$ 2001, 25:103-114.

12. Marton ML, Cordts S, Broadhvest J, Dresselhaus T: Micropylar pollen tube guidance by egg apparatus 1 of maize. Science 2005, 307:573-576.

13. Punwani JA, Drews GN: Development and function of the synergid cell. Sex Plant Reprod 2008, 21:7-15.

14. Russell SD: Double Fertilization. Int Rev Cytol 1992, 140:357-388.

15. Christensen CA, Gorsich SW, Brown RH, Jones LG, Brown J, Shaw JM, Drews GN: Mitochondrial GFA2 is required for synergid cell death in Arabidopsis. Plant Cell 2002, 14:2215-2232.

16. Rotman N, Rozier F, Boavida L, Dumas C, Berger F, Faure JE: Female control of male gamete delivery during fertilization in Arabidopsis thaliana. Curr Biol 2003, 13:432-436.

17. Fu Y, Yuan M, Huang BQ, Yang HY, Zee SY, O'Brien TP: Sex Plant Reprod 2000, 12:315-322. 
18. Russell SD: Attraction and transport of male gametes for fertilization. Sex Plant Reprod 1996, 9:337-342.

19. Sandaklie-Nikolova L, Palanivelu R, King EJ, Copenhaver GP, Drews GN: Synergid cell death in Arabidopsis is triggered following direct interaction with the pollen tube. Plant Physiol 2007, 144:1753-1762.

- 20. Hamamura Y, Saito C, Awai C, Kurihara D, Miyawaki A, Nakagawa T, Kanaoka MM, Sasaki N, Nakano A, Berger F, et al.: Live-cell imaging reveals the dynamics of two sperm cells during double fertilization in Arabidopsis thaliana. Curr Biol 2011, 21:497-502.

The authors use an innovative live-imaging technique to observe PT reception in Arabidopsis. They show the timing of PT rupture after entry into the synergids and follow the sperm as they are targeted to the egg and central cell.

21. Tsukamoto T, Qin Y, Huang Y, Dunatunga D, Palanivelu R: A role for LORELEI, a putative glycosylphosphatidylinositol-anchored protein, in Arabidopsis thaliana double fertilization and early seed development. Plant J 2010, 62:571-588.

22. Boisson-Dernier A, Frietsch S, Kim TH, Dizon MB, Schroeder JI: The peroxin loss-of-function mutation abstinence by mutual consent disrupts malefemale gametophyte recognition. Curr Biol 2008, 18:63-68.

23. Huck N, Moore JM, Federer M, Grossniklaus U: The Arabidopsis mutant feronia disrupts the female gametophytic control of pollen tube reception. Development 2003, 130:2149-2159.

24. Mansfield SG, Briarty LG: Early embryogenesis in Arabidopsis thaliana. II. The developing embryo. Can. J. Bot. 1991, 69:461-476.

25. Escobar-Restrepo JM, Huck N, Kessler S, Gagliardini V, Gheyselinck J, Yang WC, Grossniklaus U: The FERONIA receptor-like kinase mediates malefemale interactions during pollen tube reception. Science 2007, 317:656660.

26. Capron A, Gourgues M, Neiva LS, Faure JE, Berger F, Pagnussat G, Krishnan A, Alvarez-Mejia C, Vielle-Calzada JP, Lee YR, et al.: Maternal Control of Male-Gamete Delivery in Arabidopsis Involves a Putative GPI-Anchored Protein Encoded by the LORELEI Gene. Plant Cell 2008, 20:3038-3049.

$\bullet \bullet 27$. Kessler SA, Shimosato-Asano H, Keinath NF, Wuest SE, Ingram G, Panstruga $\mathrm{R}$, Grossniklaus U: Conserved molecular components for pollen tube reception and fungal invasion. Science 2010, 330:968-971.

In this paper, NORTIA, a new member of the PT reception pathway is described. NTA is a member of the MLO-family of genes originally discovered in the context of powdery mildew susceptibility. It displays a FERONIA-dependent relocalization to the filiform apparatus at PT arrival. The identification of an MLO involved in PT reception led to the discovery of a dual role for FERONIA in PT reception and powdery mildew infection, indicating that PT reception and infection may share common signaling pathways.

28. Dresselhaus T, Lausser A, Marton ML: Using maize as a model to study pollen tube growth and guidance, cross-incompatibility and sperm delivery in grasses. Ann Bot 2011.

••29. Amien S, Kliwer I, Marton ML, Debener T, Geiger D, Becker D, Dresselhaus T: Defensin-like ZmES4 mediates pollen tube burst in maize via opening of the potassium channel KZM1. PLoS Biol 2010, 8:e1000388. 
The authors describe the identification of ZmES4, a small defensin-like peptide that is secreted to the region of the filiform apparatus during PT reception. It is a species-specific mediator of $\mathrm{PT}$ rupture in in vitro germinated PTs and activates a potassium channel known as KZM1, providing an intriguing hypothesis for the control of the final stage of PT reception: the physical rupturing of the PT.

••30. Boisson-Dernier A, Roy S, Kritsas K, Grobei MA, Jaciubek M, Schroeder JI, Grossniklaus U: Disruption of the pollen-expressed FERONIA homologs ANXUR1 and ANXUR2 triggers pollen tube discharge. Development 2009, 136:3279-3288.

In this paper and the following reference, the authors describe the identification of ANXUR1/2, duplicate genes that are closely related to the FERONIA receptor like kinase. They are pollen-specific and localize to the tip of the growing pollen tube. anxl/anx2 double mutant PTs rupture prematurely in the transmitting tract, indicating that they play a role in the maintenance of PT integrity during tip growth.

••31. Miyazaki S, Murata T, Sakurai-Ozato N, Kubo M, Demura T, Fukuda H, Hasebe M: ANXUR1 and 2, sister genes to FERONIA/SIRENE, are male factors for coordinated fertilization. Curr Biol 2009, 19:1327-1331.

32. Schiott M, Romanowsky SM, Baekgaard L, Jakobsen MK, Palmgren MG, Harper JF: A plant plasma membrane Ca2+ pump is required for normal pollen tube growth and fertilization. Proc Natl Acad Sci U S A 2004, 101:95029507.

33. Coppinger P, Repetti PP, Day B, Dahlbeck D, Mehlert A, Staskawicz BJ: Overexpression of the plasma membrane-localized NDR1 protein results in enhanced bacterial disease resistance in Arabidopsis thaliana. Plant $J$ 2004, 40:225-237.

-34. Boisson-Dernier A, Kessler SA, Grossniklaus U: The walls have ears: the role of plant CrRLK1Ls in sensing and transducing extracellular signals. $J$ Exp Bot 2010, 62:1581-1591.

In this review, the authors describe the multiple functions that have been attributed to FERONIA and FER-like receptor-like kinases. They identify malectin-like carbohydrate domains in the extracellular domains of most members of this gene family and propose that these RLKs may bind carbohydrate-based ligands.

35. Huang B-Q, Russell SD, Strout GW, Mao L-G: Organization of isolated embryo sac and eggs of Plumbago zeylanica (Plumbaginaceae) before and after fertilization. Am J Bot 1990, 77:1401-1410.

36. Treub M: Sure les Casuarinées et leur place dnas le systeme naturel. Ann. Jard. Bot. Buitenzorg 1891, 10:145-231.

37. Maheswari P: An Introduction to the Embryology of Angiosperms. New Delhi: Tata McGraw-Hi; Publishing Company; 1950. 


\section{Figure Captions}

Figure 1. Pollen tube reception. (a) Pollen (yellow) germinates on a receptive stigma and extends by tip growth through the transmitting tract and one pollen tube (PT) is attracted to each ovule. (b) The mature female gametophyte has two synergid cells (white) at the micropylar end of the ovule, one egg cell (purple), and a central cell (red). (c) The PT enters the receptive synergid (gray). (d) The PT bursts and releases the two sperm cells (orange) so that double fertilization can occur. (e-f) Confocal laser scanning micrographs of PT reception. (e) PT reception in a wild-type ovule: the PT (green) enters the synergid (outlined in white) and bursts. (f) PT reception in a feronia mutant ovule. The PT is attracted normally to the synergid but fails to stop growing and burst after entry.

Figure 2. Signaling during PT reception. (a) Before PT arrival, FER and LRE are localized in the filiform apparatus, while NTA and ZmES4 are localized in unknown cytoplasmic compartments that may be part of the endomembrane system. ANX1/2 activity in the PT maintains tip growth. (b) After PT arrival at the receptive synergid, the FER pathway is activated by an unknown ligand followed by relocalization of NTA and ZmES4 to the filiform apparatus, although it is currently not known whether this is a direct or indirect mechanism. Feedback to ANX1/2 leads to PT rupture, perhaps through modulation of ion influx mediated by $\mathrm{ZmES} 4$ control of the $\mathrm{KZm} 4 \mathrm{~K}^{+}$channel and/or $\mathrm{Ca}^{2+}$ through the ACA9 channel. 
Figure 1
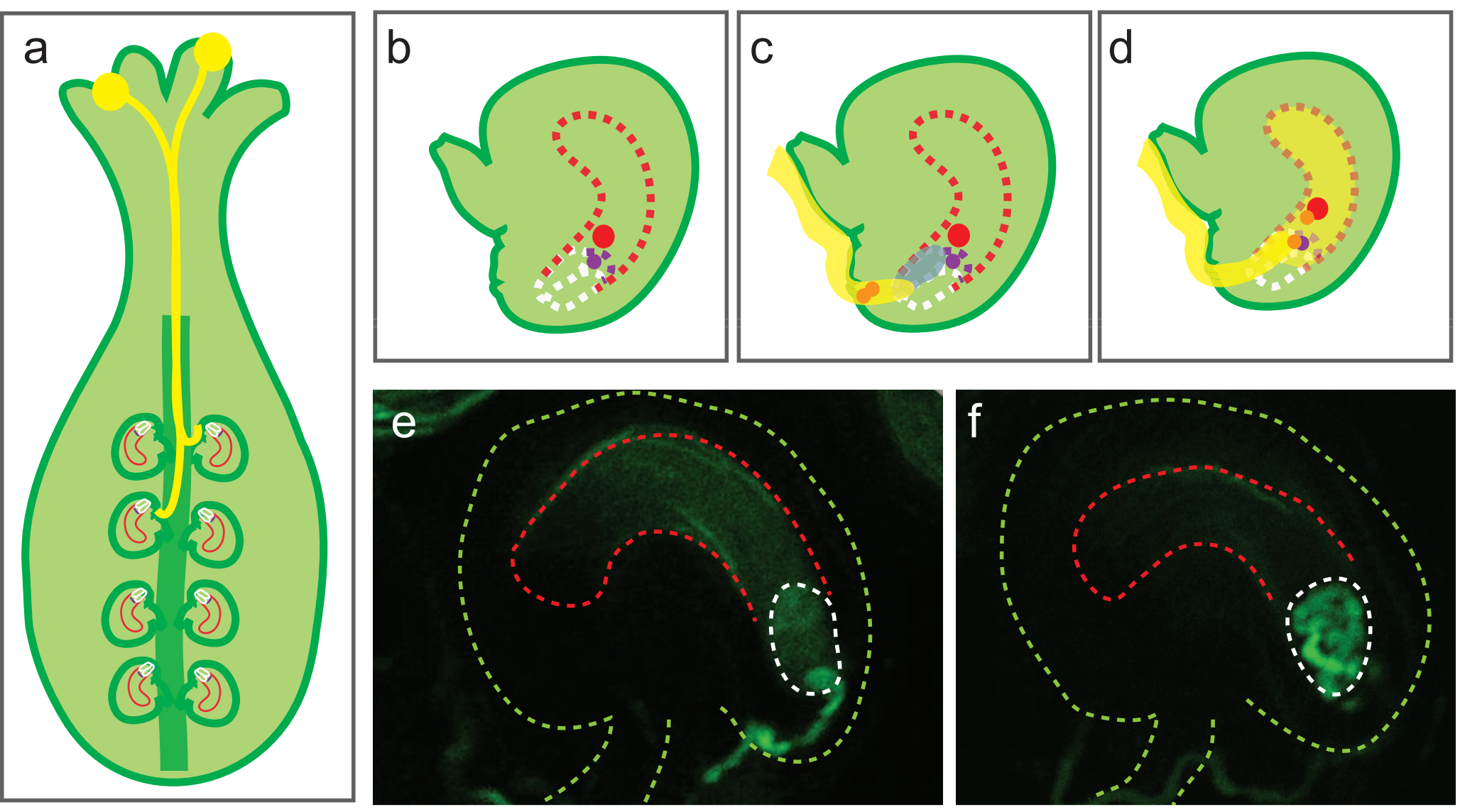
Figure 2

Figure 2

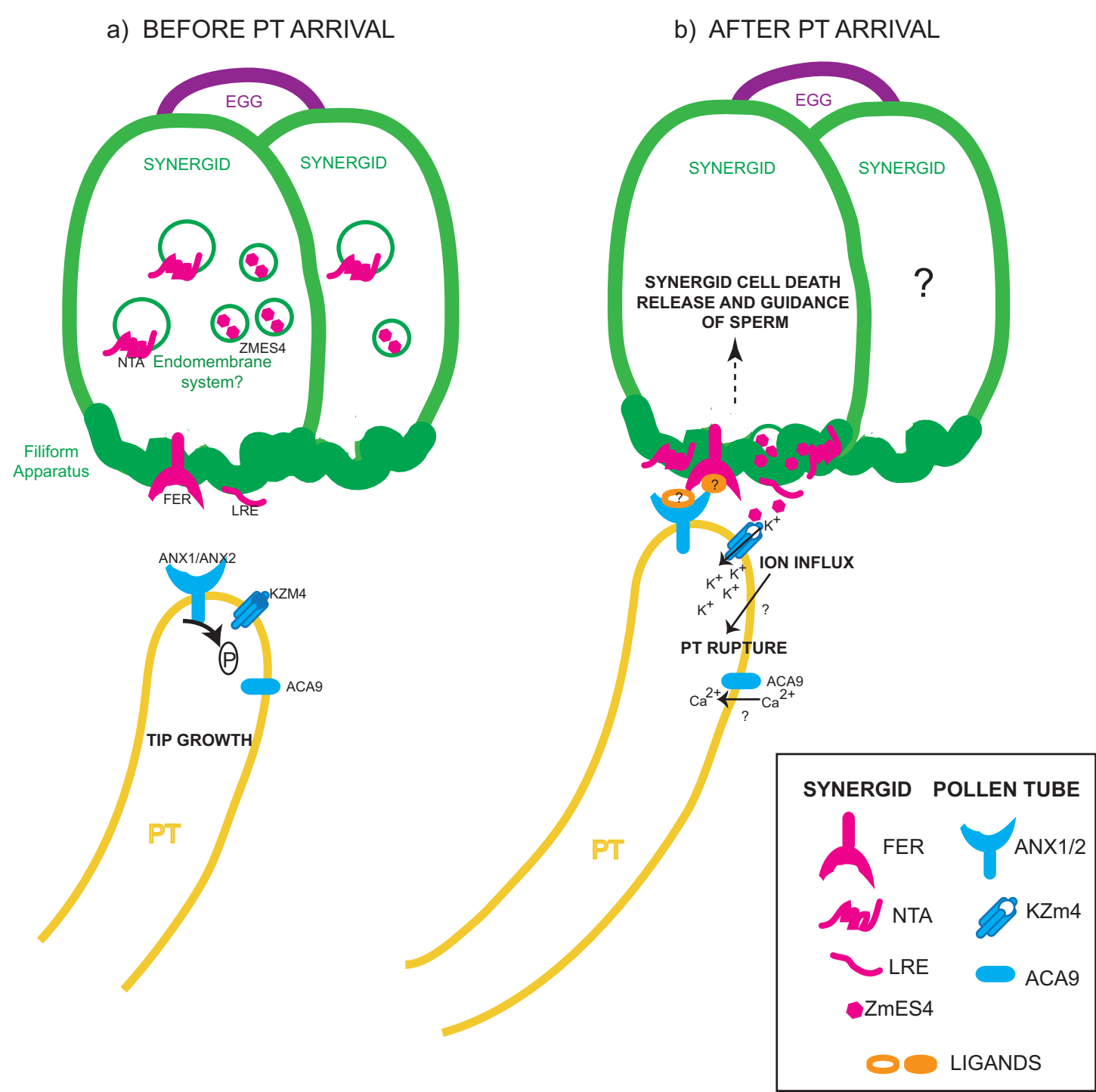

